Pure and Applied Mathematics Quarterly

Volume 6, Number 3

(Special Issue: In honor of

Joseph J. Kohn, Part 1 of 2)

$829-842,2010$

\title{
Polynomial and Rational Maps between Balls
}

James Faran, Xiaojun Huang*, Shanyu Ji and Yuan Zhang

Dedicated to Professor J.J.Kohn on the occasion of his 75th birthday

\begin{abstract}
For a proper holomorphic map $F$ from a ball $\mathbb{B}^{n}$ into another ball $\mathbb{B}^{N}$, we give a criterion when a rational map $F$ is equivalent to a polynomial one. As application, we show that proper rational holomorphic maps from $\mathbb{B}^{2}$ into $\mathbb{B}^{N}$ of degree two are equivalent to polynomial maps, and we also give an example of a rational holomorphic maps of degree 3 that are 'almost' linear but are not equivalent to holomorphic polynomial maps.
\end{abstract}

Keywords: proper holomorphic maps, maps between balls, rational maps, polynomial maps, criterion, equivalent

\section{INTRODUCTION}

Let $\mathbb{B}^{n}$ be the unit ball in the complex space $\mathbb{C}^{n}$. Write $\operatorname{Rat}\left(\mathbb{B}^{n}, \mathbb{B}^{N}\right)$ for the space of proper rational holomorphic maps from $\mathbb{B}^{n}$ into $\mathbb{B}^{N}$ and Poly $\left(\mathbb{B}^{n}, \mathbb{B}^{N}\right)$ for the set of proper holomorphic polynomial maps from $\mathbb{B}^{n}$ into $\mathbb{B}^{N}$. We say that $F$ and $G \in \operatorname{Rat}\left(\mathbb{B}^{n}, \mathbb{B}^{N}\right)$ are equivalent if there are automorphisms $\sigma \in \operatorname{Aut}\left(\mathbb{B}^{n}\right)$ and $\tau \in \operatorname{Aut}\left(\mathbb{B}^{N}\right)$ such that $F=\tau \circ G \circ \sigma$.

Proper holomorphic maps from $\mathbb{B}^{n}$ into $\mathbb{B}^{N}$ with $N \leq 2 n-2$, that are sufficiently smooth up to the boundary, are equivalent to the identity map ([Fa] $[\mathrm{Fr}]$ $[\mathrm{Hu}])$. In [HJX], it is shown that $F \in \operatorname{Rat}\left(\mathbb{B}^{n}, \mathbb{B}^{N}\right)$ with $N \leq 3 n-4$ is equivalent to a quadratic monomial map, called the D'Angelo map. However, when the

Received Octomber 24, 2007.

*Supported in part by NSF-0801056 
codimension is sufficiently large, there is plenty of room to construct rational holomorphic maps with certain arbitrariness by the work in Catlin-D'Angelo [CD]. Hence, it is reasonable to believe that after lifting the codimension restriction, many proper rational holomorphic maps are not equivalent to proper holomorphic polynomial maps. In the last paragraph of the paper [DA], D'Angelo gave a philosophic discussion on this matter. However, the problem of determining if an explicit proper rational holomorphic map is equivalent to a polynomial map does not seem to have been studied so far.

This short paper is concerned with such a problem. We will first give a simple and explicit criterion when a rational holomorphic map is equivalent to a holomorphic polynomial map. With the help of the classification result in [CJX], this criterion is used in $\S 3$ to show that proper rational holomorphic maps from $\mathbb{B}^{2}$ into $\mathbb{B}^{N}$ of degree two are equivalent to polynomial maps. On the other hand, making use of the criterion, we construct in $\S 4$ rational holomorphic maps of degree 3 that are 'almost' linear but are not equivalent to holomorphic polynomial maps.

Acknowledgment: This work was started when the second and the third authors were visiting the School of Mathematics, Wuhan University, China, in the summer of 2005. They are indebted to Professor Hua Chen for his support, arrangement and hospitality, which made the visit possible. The authors of the paper also thank J. D'Angelo and P. Ebenfelt for the invitation to the AIM workshop on Complexity of Mappings in CR Geometry in September, 2006. The fourth author would like to thank F. Meylan and D. Zaitsev for discussions related to the paper.

\section{A CRITERION}

Let $F=\frac{P}{q}=\frac{\left(P_{1}, \ldots, P_{N}\right)}{q}$ be a non-constant rational holomorphic map from the unit ball $\mathbb{B}^{n} \subset \mathbb{C}^{n}$ into the unit ball $\mathbb{B}^{N} \subset \mathbb{C}^{N}$, where $\left(P_{j}\right)_{j=1}^{N}$, q are holomorphic polynomial functions and $\left(P_{1}, \ldots, P_{N}, q\right)=1$. We define $\operatorname{deg}(F)=$ $\max \left\{\operatorname{deg}\left(P_{j}\right)_{j=1}^{N}, \operatorname{deg}(q)\right\}$. Then $F$ induces a rational map from $\mathbb{C P}^{n}$ into $\mathbb{C P}^{N}$ given by

$$
\hat{F}\left(\left[z_{1}: \cdots: z_{n}: t\right]\right)=\left[t^{k} P\left(\frac{z}{t}\right): t^{k} q\left(\frac{z}{t}\right)\right]
$$

where $z=\left(z_{1}, \ldots, z_{n}\right) \in \mathbb{C}^{n}$ and $\operatorname{deg}(F)=k>0$. 
$\hat{F}$ may not be holomorphic in general. Denote by $\operatorname{Sing}(\hat{F})$ the singular set of $\hat{F}$, namely, the collection of points where $\hat{F}$ fails to be (or fails to extend to be) holomorphic. Then $\operatorname{Sing}(\hat{F})$ is an algebraic subvariety of codimension two or more in $\mathbb{C P}^{n}$. For instance, we have the following:

\section{Example 2.1}

I. Let $F_{\theta}(z, w)=\left(z, \cos \theta w, \sin \theta z w, \sin \theta w^{2}\right)$ be the proper monomial map from $\mathbb{B}^{2}$ into $\mathbb{B}^{4}$ (called the D'Angelo map), where $0<\theta<\frac{\pi}{2}$. Then $\operatorname{Sing}\left(\hat{F}_{\theta}\right)$ of $\hat{F}_{\theta}$ consists of one point: $\left\{[z: w: t] \in \mathbb{C P}^{2}: w=0, t=0\right\}=\{[1: 0: 0]\}$.

II. Let $G_{\alpha}=\left(z^{2}, \sqrt{1+\cos ^{2} \alpha} z w, \cos \alpha w^{2}, \sin \alpha w\right)$ be the proper monomial map from $\mathbb{B}^{2}$ into $\mathbb{B}^{4}$ where $0 \leq \alpha<\frac{\pi}{2}$. Then $G_{\alpha}$ induces

$$
\hat{G}_{\alpha}=\left[z^{2}: \sqrt{1+\cos ^{2} \alpha} z w: \cos \alpha w^{2}: \sin \alpha w t: t^{2}\right] .
$$

There are no singular points for $\hat{G}_{\alpha}$. Hence $\hat{G}_{\alpha}$ is holomorphic.

Write $\mathbb{B}_{1}^{n}=\left\{\left[z_{1}: \cdots: z_{n}: t\right] \in \mathbb{C P}^{n}: \sum_{j=1}^{n}\left|z_{j}\right|^{2}<|t|^{2}\right\}$, which is the projective realization of $\mathbb{B}^{n}$. Write $U(n+1,1)$ for the collection of the linear transforms $\mathcal{A}:[Z]\left(\in \mathbb{C P}^{n}\right) \mapsto[Z A]\left(\in \mathbb{C P}^{n}\right)$ such that

$$
A E_{n+1,1} \bar{A}^{t}=E_{n+1,1}
$$

where

$$
E_{n+1,1}=\left(\begin{array}{cc}
I_{n} & 0 \\
0 & -1
\end{array}\right) .
$$

Then $U(n+1,1) /\{ \pm I d\}=\operatorname{Aut}\left(\mathbb{B}_{1}^{n}\right) \approx \operatorname{Aut}\left(\mathbb{B}^{n}\right)$.

Lemma 2.1. For any hyperplane $H \subset \mathbb{C P}^{n}$ with $H \cap \overline{\mathbb{B}}_{1}^{n}=\emptyset$, there is a $\sigma \in$ $U(n+1,1)$ such that $\sigma(H)=H_{\infty}=\left\{\left[z_{1}: \cdots: z_{n}: 0\right] \in \mathbb{C P}^{n}\right\}$.

Proof: Assume that $H: \sum_{j=1}^{n} a_{j} z_{j}-a_{n+1} t=0$ with $\vec{a}=\left(a_{1}, \ldots, a_{n+1}\right) \neq 0$. Under the assumption that $H \cap \overline{\mathbb{B}_{1}^{n}}=\emptyset$, we have $a_{n+1} \neq 0$. Without loss of generality, we can assume that $a_{n+1}=1$. Let $U$ be an $n \times n$ unitary matrix such that

$$
\left(a_{1}, \ldots, a_{n}\right) \bar{U}=(\lambda, 0, \ldots, 0)
$$


for some $\lambda \in \mathbb{C}$. Let $\sigma=\left(\begin{array}{ll}U & 0 \\ 0 & I\end{array}\right)$. Then $\sigma(H)=\left\{[z: t] \in \mathbb{C P}^{n} \mid \lambda z_{1}-t=0\right\}$ with $|\lambda|<1$. Let $T \in A u t\left(\mathbb{B}^{n}\right)$ be defined by

$$
T\left(z_{1}, z^{\prime}\right)=\left(\frac{z_{1}-\bar{\lambda}}{1-\lambda z_{1}}, \frac{\sqrt{1-|\lambda|^{2}} z^{\prime}}{1-\lambda z_{1}}\right)
$$

with $z^{\prime}=\left(z_{2}, \ldots, z_{n}\right)$. Then $\hat{T} \in U(n+1,1)$ is defined by

$$
\hat{T}\left(\left[z_{1}: z^{\prime}: t\right]\right)=\left[z_{1}-\bar{\lambda} t: \sqrt{1-|\lambda|^{2}} z^{\prime}: t-\lambda z_{1}\right] .
$$

Now, it is easy to see that $\hat{T} \circ \sigma$ maps $H$ to $H_{\infty}$.

Our criterion can be stated as follows:

Theorem 2.2. Let $F$ be a non-constant rational holomorphic map from $\mathbb{B}^{n}$ into $\mathbb{B}^{N}$ with $N, n \geq 1$. Then $F$ is equivalent to a holomorphic polynomial map from $\mathbb{B}^{n}$ into $\mathbb{B}^{N}$, namely, there are $\sigma \in \operatorname{Aut}\left(\mathbb{B}^{n}\right)$ and $\tau \in \operatorname{Aut}\left(\mathbb{B}^{N}\right)$ such that $\tau \circ F \circ \sigma$ is a holomorphic polynomial map from $\mathbb{B}^{n}$ into $\mathbb{B}^{N}$, if and only if there exist (complex) hyperplanes $H \subset \mathbb{C P}^{n}$ and $H^{\prime} \subset \mathbb{C P}^{N}$ such that $H \cap \overline{\mathbb{B}_{1}^{n}}=\emptyset$, $H^{\prime} \cap \overline{\mathbb{B}_{1}^{N}}=\emptyset$ and

$$
\hat{F}(H \backslash \operatorname{Sing}(\hat{F})) \subset H^{\prime}, \quad \hat{F}\left(\mathbb{C P}^{n} \backslash(H \cup \operatorname{Sing}(\hat{F}))\right) \subset \mathbb{C P}^{N} \backslash H^{\prime} .
$$

Proof: If $F$ is a non-constant holomorphic polynomial map, then $\hat{F}=\left[t^{k} F\left(\frac{z}{t}\right), t^{k}\right]$ with $\operatorname{deg}(F)=k>0$. Let $H=H_{\infty}$ and $H^{\prime}=H_{\infty}^{\prime}$. Then they satisfy the property described in the theorem.

If $F$ is equivalent to a holomorphic polynomial map $G$, then there exist $\hat{\sigma} \in$ $U(n+1,1), \hat{\tau} \in U(n+1,1)$ such that $\hat{F}=\hat{\tau} \circ \hat{G} \circ \hat{\sigma}$. Let $H=\hat{\sigma}^{-1}\left(H_{\infty}\right)$ and $H^{\prime}=\hat{\tau}\left(H_{\infty}^{\prime}\right)$. Then they are the desired ones.

Conversely, suppose that $\hat{F}, H$ and $H^{\prime}$ are as in the theorem. By Lemma 2.1, we can find $\hat{\sigma} \in U(n+1,1)$ and $\hat{\tau} \in U(n+1,1)$ such that $\hat{\sigma}(H)=H_{\infty}$ and $\hat{\tau}\left(H^{\prime}\right)=H_{\infty}^{\prime}$. Let $\hat{Q}=\hat{\tau} \circ \hat{F} \circ \hat{\sigma}^{-1}$. Then $\hat{Q}$ induces a rational holomorphic map $Q$ from $\mathbb{B}^{n}$ into $\mathbb{B}^{N}$. If $Q=\frac{P}{q}$ where $(P, q)=1$ and $\operatorname{deg}(Q)=k>0$, then

$$
\hat{Q}=\left[t^{k} P\left(\frac{z}{t}\right): t^{k} q\left(\frac{z}{t}\right)\right]
$$

Suppose that $q \not \equiv$ constant. Let $z_{0} \in \mathbb{C}^{n}$ be such that $q\left(z_{0}\right)=0$ but $P\left(z_{0}\right) \neq 0$. Then $\left[z_{0}: 1\right] \notin \operatorname{Sing}(\hat{Q}) \cup H_{\infty}$ and $\hat{Q}\left(\left[z_{0}: 1\right]\right) \in H_{\infty}^{\prime}$. Notice that $\hat{Q}\left(H_{\infty} \backslash\right.$ 
$\operatorname{Sing}(\hat{Q})) \subset H_{\infty}^{\prime}$ and $\hat{Q}\left(\mathbb{C P}^{n} \backslash\left(H_{\infty} \cup \operatorname{Sing}(\hat{Q})\right)\right) \subset \mathbb{C P}^{N} \backslash H_{\infty}^{\prime}$. This is a contradiction. Thus, we showed that $Q$ is a polynomial.

Remark 2.3 (A): Suppose that $\hat{F}=\left[F_{1}: \cdots: F_{N}: F_{0}\right]$ is a non-constant rational map from $\mathbb{C P}^{n}$ into $\mathbb{C P}^{N}$, where $F_{0}, \ldots, F_{N}$ are homogeneous polynomials in $(z, t)$ of degree $k>0$ with

$$
\left(F_{1}, \ldots, F_{N}, F_{0}\right)=1 .
$$

Assume that $H:=\left\{\left[z_{1}: \cdots: z_{n}: t\right] \in \mathbb{C P}^{n}: \sum_{j=1}^{n} a_{j} z_{j}+a_{0} t=0, a_{j} \in\right.$ $\left.\mathbb{C},\left(a_{0}, \ldots, a_{n}, a_{0}\right) \neq 0\right\}, H^{\prime}:=\left\{\left[z_{1}^{\prime}: \cdots: z_{N}^{\prime}: t^{\prime}\right] \in \mathbb{C P}^{N}: \sum_{j=1}^{N} A_{j} z_{j}^{\prime}+A_{0} t^{\prime}=\right.$ $\left.0, A_{j} \in \mathbb{C},\left(A_{0}, \ldots, A_{N}, A_{0}\right) \neq 0\right\}$ are (complex) hyperplanes. Also assume that $H \cap \mathbb{B}_{1}^{n}=\emptyset$ and $H^{\prime} \cap \mathbb{B}_{1}^{N}=\emptyset$. We easily see that $a_{0}, A_{0} \neq 0$ ( thus we can always make $a_{0}, A_{0}=1$ ). Under such a set-up, by the basic division property for polynomials, we can easily conclude that

$$
\hat{F}(H \backslash \operatorname{Sing}(\hat{F})) \subset H^{\prime}, \quad \hat{F}\left(\mathbb{C P}^{n} \backslash(H \cup \operatorname{Sing}(\hat{F}))\right) \subset \mathbb{C P}^{N} \backslash H^{\prime}
$$

if and only if

$$
\sum_{j=1}^{N} A_{j} F_{j}+A_{0} F_{0} \equiv C \cdot\left(\sum_{j=1}^{n} a_{j} z_{j}+a_{0} t\right)^{k},
$$

where $C \neq 0$ is a constant and $k(>0)$ is the degree of $F$. This observation will be used for our later application of Theorem 2.2.

(B): From the argument of Theorem 2.2, it is clear that a similar result can also be proved for non-constant rational maps from $\mathbb{C P}^{n}$ into $\mathbb{C P}^{N}$.

Write the Cayley transformation

$$
\rho_{n}\left(z^{\prime}, z_{n}\right)=\left(\frac{2 z^{\prime}}{1-i z_{n}}, \frac{1+i z_{n}}{1-i z_{n}}\right) .
$$

Then $\rho_{n}$ biholomorphically maps $\partial \mathbb{H}^{n}$ to $\partial \mathbb{B}^{n} \backslash\{(0,1)\}$, where $\mathbb{H}^{n}=\left\{\left(z^{\prime}, z_{n}\right) \in\right.$ $\left.\mathbb{C}^{n}: \Im\left(z_{n}\right)>\left|z^{\prime}\right|^{2}\right\} . \rho_{n}$ induces a linear transformation of $\mathbb{C P}^{n}$ :

$$
\hat{\rho}_{n}=\left[2 z^{\prime}: t+i z_{n}: t-i z_{n}\right] .
$$

$\hat{\rho}_{n}$ maps $\mathbf{S}_{1}^{n}=\left\{\left[z^{\prime}: z_{n}: t\right] \in \mathbb{C P}^{n}: \frac{z_{n} \bar{t}-t \bar{z}_{n}}{2 i}>\left|z^{\prime}\right|^{2}\right\}$ to $\mathbb{B}_{1}^{n}$.

Now let $G$ be a non-constant rational holomorphic map from an open piece of $\partial \mathbb{H}^{n}$ into $\partial \mathbb{H}^{N}$. Then $\rho_{N} \circ G \circ \rho_{n}^{-1}$ extends to a proper rational holomorphic map from $\mathbb{B}^{n}$ to $\mathbb{B}^{N}$. By Theorem 2.2, we have the following: 
Theorem $2.4 \rho_{N} \circ G \circ \rho_{n}^{-1}$ is equivalent to a proper holomorphic polynomial map from $\mathbb{B}^{n}$ into $\mathbb{B}^{N}$ if and only if there are (complex) hyperplanes $H \subset \mathbb{C P}^{n}$, $H^{\prime} \subset \mathbb{C P}^{N}$ such that $\hat{G}(H \backslash \operatorname{Sing}(\hat{G})) \subset H^{\prime}$ and $\hat{G}\left(\mathbb{C P}^{n} \backslash(H \cup \operatorname{Sing}(\hat{G})) \subset \mathbb{C P}^{N} \backslash H^{\prime}\right.$ with

$$
H \cap \overline{\mathbf{S}_{1}^{n}}=\emptyset, \quad H^{\prime} \cap \overline{\mathbf{S}_{1}^{N}}=\emptyset
$$

3. Proper rational holomorphic maps from $\mathbb{B}^{2}$ into $\mathbb{B}^{N}$ of Degree

TWO

As a first application of Theorem 2.2, we prove the following:

Theorem 3.1. A map $F \in \operatorname{Rat}\left(\mathbb{B}^{2}, \mathbb{B}^{N}\right)$ of degree two is equivalent to a polynomial proper holomorphic map in Poly $\left(\mathbb{B}^{2}, \mathbb{B}^{N}\right)$.

Proof: By [HJX], we know that any rational holomorphic map of degree 2 from $\mathbb{B}^{2}$ into $\mathbb{B}^{N}$ is equivalent to a map of the form $(G, 0)$, where the map $G$ is from $\mathbb{B}^{2}$ into $\mathbb{B}^{5}$. Hence, to prove Theorem 3.1, we need only to assume that $N=5$. After applying Cayley transformations and using the result in [CJX], we can assume that $F=\left(f, \phi_{1}, \phi_{2}, \phi_{3}, g\right)$ is from $\mathbb{H}^{2}$ into $\mathbb{H}^{5}$ with either

(I)

$$
f=\frac{z+\frac{i}{2} z w}{1+e_{2} w^{2}}, \phi_{1}=\frac{z^{2}}{1+e_{2} w^{2}}, \phi_{2}=\frac{c_{1} z w}{1+e_{2} w^{2}}, \phi_{3}=0, g=\frac{w}{1+e_{2} w^{2}}
$$

where $-e_{2}=\frac{1}{4}+c_{1}^{2}$ and $c_{1}>0$ or

$$
\begin{gathered}
f=\frac{z+\left(\frac{i}{2}+i e_{1}\right) z w}{1+i e_{1} w+e_{2} w^{2}}, \phi_{1}=\frac{z^{2}}{1+i e_{1} w+e_{2} w^{2}}, \\
\phi_{2}=\frac{c_{1} z w}{1+i e_{1} w+e_{2} w^{2}}, \phi_{3}=\frac{c_{3} w^{2}}{1+i e_{1} w+e_{2} w^{2}}, g=\frac{w+i e_{1} w^{2}}{1+i e_{1} w+e_{2} w^{2}}
\end{gathered}
$$

where $-e_{1},-e_{2}>0, c_{1}, c_{3}>0$ and

$$
e_{1} e_{2}=c_{3}^{2}, \quad-e_{1}-e_{2}=\frac{1}{4}+c_{1}^{2} .
$$

Write $[z: w: t]$ for the homogeneous coordinates of $\mathbb{C P}^{2}$. In Case (I) the map $F$ induces a rational map $\hat{F}: \mathbb{C P}^{2} \rightarrow \mathbb{C P}^{5}$ given by

$$
\hat{F}([z: w: t])=\left[t z+\frac{i}{2} z w: z^{2}: c_{1} z w: 0: t w: t^{2}+e_{2} w^{2}\right] \quad \forall[z: w: t] \in \mathbb{C P}^{2} .
$$


In Case (II), $F$ induces a rational map $\hat{F}: \mathbb{C P}^{2} \rightarrow \mathbb{C P}^{5}$ given by $\hat{F}([z: w: t])=\left[t z+\left(\frac{i}{2}+i e_{1}\right) z w: z^{2}: c_{1} z w: c_{3} w^{2}: t w+i e_{1} w^{2}: t^{2}+i e_{1} w t+e_{2} w^{2}\right]$ $\forall[z: w: t] \in \mathbb{C P}^{2}$. In terms of Theorem 2.4, we will look for $H=\{-t=$ $\left.\mu_{1} z_{1}+\mu_{2} z_{2}\right\} \subset \mathbb{C P}^{2}$ and $H^{\prime}=\left\{-t^{\prime}=\sum_{j=1}^{5} \lambda_{j} z_{j}^{\prime}\right\} \subset \mathbb{C P}^{5}$ such that $H \cap \overline{\mathbf{S}_{1}^{2}}=$ $\emptyset, \quad H^{\prime} \cap \overline{\mathbf{S}_{1}^{5}}=\emptyset$ with

$$
\hat{F}(H \backslash \operatorname{Sing}(\hat{F})) \subset H^{\prime} \text { and } \hat{F}\left(\mathbb{C P}^{2} \backslash(H \cup \operatorname{Sing}(\hat{F}))\right) \subset \mathbb{C P}^{5} \backslash H^{\prime} .
$$

We next prove the following lemma:

Lemma 3.2. Let $H=\left\{-t=\sum_{j=1}^{n} K_{j} z_{j}\right\} \subset \mathbb{C P}^{n}$. Then $H \cap \overline{\mathbf{S}_{1}^{n}}=\emptyset$ if and only if

$$
4 \Im\left(K_{n}\right)+\sum_{j=1}^{n-1}\left|K_{j}\right|^{2}<0 .
$$

Proof: $\quad$ Suppose for $z_{j}$ and $t=-\sum_{j=1}^{n} K_{j} z_{j}$, we have

$$
\frac{w \bar{t}-t \bar{w}}{2 i}<\sum_{j=1}^{n-1}\left|z_{j}\right|^{2}
$$

Here we identify $z_{n}=w$. We then get

$$
\frac{-\overline{K_{n}}|w|^{2}+K_{n}|w|^{2}}{2 i}+\sum_{j=1}^{n-1} \frac{-\overline{K_{j}} \overline{z_{j}} w+K_{j} z_{j} \bar{w}}{2 i}<\sum_{j=1}^{n-1}\left|z_{j}\right|^{2} .
$$

Hence

$$
|w|^{2} \Im\left(K_{n}\right)<\sum_{j=1}^{n-1}\left\{\left|z_{j}\right|^{2}-2 \Re\left(\frac{K_{j}}{2 i} z_{j} \bar{w}\right)\right\},
$$

or

$$
|w|^{2}\left(\Im\left(K_{n}\right)+\sum_{j=1}^{n-1} \frac{\left|K_{j}\right|^{2}}{4}\right)<\sum_{j=1}^{n-1}\left|z_{j}-\frac{i}{2} \overline{K_{j}} w\right|^{2} .
$$

Since $\left\{z_{j}, w\right\}$ are independent variables, this can only happen if and only if

$$
\Im\left(K_{n}\right)+\sum_{j=1}^{n-1} \frac{\left|K_{j}\right|^{2}}{4}<0 .
$$

This proves the lemma. 
We first consider Case (I). Here, we need only to find out $\mu_{1}, \mu_{2}, \lambda_{1}, \ldots, \lambda_{5} \in \mathbb{C}$ such that

$$
4 \Im\left(\mu_{2}\right)+\left|\mu_{1}\right|^{2}<0, \quad 4 \Im\left(\lambda_{5}\right)+\sum_{j=1}^{4}\left|\lambda_{j}\right|^{2}<0
$$

and

$$
\lambda_{1}\left(t z+\frac{i}{2} z w\right)+\lambda_{2} z^{2}+\lambda_{3} c_{1} z w+\lambda_{5} t w+\left(t^{2}+e_{2} w^{2}\right)=\left(t+\mu_{1} z+\mu_{2} w\right)^{2},
$$

$\forall[z: w: t] \in \mathbb{C P}^{2}$. It is easy to verify that $\lambda_{1}=\lambda_{2}=\lambda_{3}=\lambda_{4}=\mu_{1}=0$, $\lambda_{5}=-2 \sqrt{\left|e_{2}\right|} i$ and $\mu_{2}=-\sqrt{\left|e_{2}\right|} i$ satisfy the above conditions. Hence in Case (I), the map is always equivalent to a holomorphic polynomial map in Poly $\left(\mathbb{B}^{2}, \mathbb{B}^{5}\right)$.

We next consider the second case. Similar to Case (I), it suffices for us to find

$$
\mu_{1}, \mu_{2}, \lambda_{1}, \ldots, \lambda_{5} \in \mathbb{C}
$$

such that

$$
4 \Im\left(\mu_{2}\right)+\left|\mu_{1}\right|^{2}<0, \quad 4 \Im\left(\lambda_{5}\right)+\sum_{j=1}^{4}\left|\lambda_{j}\right|^{2}<0
$$

and

$$
\begin{aligned}
& \lambda_{1}\left(t z+i\left(\frac{1}{2}+e_{1}\right) z w\right)+\lambda_{2} z^{2}+\lambda_{3} c_{1} z w+\lambda_{4} c_{3} w^{2}+\lambda_{5}\left(t w+i e_{1} w^{2}\right) \\
& +\left(t^{2}+i e_{1} t w+e_{2} w^{2}\right)=\left(t+\mu_{1} z+\mu_{2} w\right)^{2}, \quad \forall[z: w: t] \in \mathbb{C P}^{2} .
\end{aligned}
$$

Comparing the coefficients, we get

$$
\begin{aligned}
& \lambda_{1}=2 \mu_{1}, \lambda_{2}=\mu_{1}^{2}, \lambda_{3}=\frac{1}{c_{1}}\left[-i\left(1+2 e_{1}\right) \mu_{1}+2 \mu_{1} \mu_{2}\right], \\
& \lambda_{4}=\frac{1}{c_{3}}\left(\mu_{2}^{2}-e_{2}-2 i e_{1} \mu_{2}-e_{1}^{2}\right), \lambda_{5}=2 \mu_{2}-i e_{1} .
\end{aligned}
$$

By Theorem 2.4 and Remark 2.3, we thus obtain the following statement:

$\rho_{N} \circ F \circ \rho_{n}^{-1}$ is equivalent to a holomorphic polynomial map if and only if there are $\mu_{1}, \mu_{2} \in \mathbb{C}$ such that $4 \Im\left(\mu_{2}\right)+\left|\mu_{1}\right|^{2}<0$ and that

$-4 e_{1}+8 \Im\left(\mu_{2}\right)+4\left|\mu_{1}\right|^{2}+\left|\mu_{1}\right|^{4}+\frac{1}{c_{1}^{2}}\left|2 \mu_{1} \mu_{2}-i\left(1+2 e_{1}\right) \mu_{1}\right|^{2}+\frac{1}{c_{3}^{2}}\left|\mu_{2}^{2}-e_{2}-e_{1}^{2}-2 i e_{1} \mu_{2}\right|^{2}<0$.

We will look for $\mu_{1}$ and $\mu_{2}$ with $\mu_{1}=0$ and $\mu_{2}=i y(y<0)$. 
To prove that $\rho_{N} \circ F \circ \rho_{n}^{-1}$ is equivalent to a polynomial map, it suffices for us to show that there exists $y<0$ such that

$$
-4 e_{1}+8 y+\frac{1}{c_{3}^{2}}\left(-y^{2}-e_{2}-e_{1}^{2}+2 e_{1} y\right)^{2}<0,
$$

or

$J(y):=\left(-4 e_{1}+8 y\right) e_{1} e_{2}+\left(y^{2}-2 e_{1} y+e_{1}^{2}+e_{2}\right)^{2}=\left(8 y-4 e_{1}\right) e_{1} e_{2}+\left(\left(y-e_{1}\right)^{2}+e_{2}\right)^{2}<0$.

Notice that as a function in $y<0$,

$$
\lim _{y \rightarrow-\infty} J(y)=+\infty, J(0)=\left(e_{1}^{2}-e_{2}\right)^{2}>0 .
$$

We need to show that

$$
\min _{y \leq 0} J(y)<0 .
$$

Notice that $J^{\prime}(y)=8 e_{1} e_{2}+4\left(\left(y-e_{1}\right)^{2}+e_{2}\right)\left(y-e_{1}\right)$. Setting $J^{\prime}(y)=0$, we get

$$
\left(y-e_{1}\right)^{3}+e_{2}\left(y-e_{1}\right)+2 e_{1} e_{2}=0 .
$$

$J^{\prime}(y)=0$ thus has a root $y_{0} \in(-\infty, 0)$; for

$$
\lim _{y \rightarrow-\infty} J^{\prime}(y)=-\infty, \quad J^{\prime}(0)=4\left(-e_{1}^{3}+e_{1} e_{2}\right)>0 .
$$

Let $\zeta_{0}, \zeta_{1}, \zeta_{2}$ be the solution of

$$
\zeta^{3}+e_{2} \zeta+2 e_{1} e_{2}=0 \text { with } \zeta_{0}=y_{0}-e_{1} .
$$

Then $\zeta_{0}+\zeta_{1}+\zeta_{2}=0, \zeta_{0} \zeta_{1}+\zeta_{0} \zeta_{2}+\zeta_{1} \zeta_{2}=e_{2}$ and $\zeta_{0} \zeta_{1} \zeta_{2}=-2 e_{1} e_{2}$. Hence $\zeta_{0}=-\zeta_{1}-\zeta_{2}$. We get

$$
-\zeta_{0}^{2}+\zeta_{1} \zeta_{2}=e_{2}
$$

or $\zeta_{1} \zeta_{2}=e_{2}+\zeta_{0}^{2}$, and

In particular, $\frac{1}{\zeta_{1} \zeta_{2}} \in \mathbb{R} \backslash\{0\}$.

$$
\frac{1}{\zeta_{1} \zeta_{2}}=-\frac{\zeta_{0}}{2 e_{1} e_{2}}
$$

Now $J\left(y_{0}\right)=\left(-4 e_{1}+8 \zeta_{0}+8 e_{1}\right) e_{1} e_{2}+\left(\zeta_{0}^{2}+e_{2}\right)^{2}=2 e_{1} e_{2}\left(4 \zeta_{0}+2 e_{1}\right)+\left(\zeta_{1} \zeta_{2}\right)^{2}$ $=-\zeta_{0} \zeta_{1} \zeta_{2}\left(4 \zeta_{0}+2 e_{1}\right)+\left(\zeta_{1} \zeta_{2}\right)^{2}$.

Notice that $4 \zeta_{0}^{3}=-8 e_{1} e_{2}-4 e_{2} \zeta_{0}$. We see that

$$
\begin{aligned}
& 2 e_{1} e_{2} \frac{J\left(y_{0}\right)}{\left(\zeta_{1} \zeta_{2}\right)^{2}}=2 e_{1} e_{2}+\zeta_{0}^{2}\left(4 \zeta_{0}+2 e_{1}\right)=2 e_{1} e_{2}-8 e_{1} e_{2}-4 e_{2} \zeta_{0}+2 e_{1} \zeta_{0}^{2} \\
& =-6 e_{1} e_{2}-4 e_{2} \zeta_{0}+2 e_{1} \zeta_{0}^{2}=-2 e_{2}\left(3 e_{1}+2 \zeta_{0}\right)+2 e_{1} \zeta_{0}^{2} .
\end{aligned}
$$


Since $\zeta_{0}=y_{0}-e_{1}<-e_{1}, 3 e_{1}+2 \zeta_{0}<e_{1}<0$. Therefore $\frac{J\left(y_{0}\right)}{\left(\zeta_{1} \zeta_{2}\right)^{2}} 2 e_{1} e_{2}<0$. Hence we showed that $J\left(y_{0}\right)<0$. This also completes the proof of Theorem 3.1.

Our proof of Theorem 3.1 is, in fact, a constructive proof, which can be used to find precisely polynomial maps equivalent to the original ones. In the following, we demonstrate this by giving an explicit example:

Proposition 3.3 Let $F=\left(f, \phi_{1}, \phi_{2}, \phi_{3}, g\right): \mathbb{H}^{2} \rightarrow \mathbb{H}^{5}$ be defined as follows:

$$
\begin{gathered}
f(z, w)=\frac{z-\frac{i}{2} z w}{1-i w-\frac{1}{3} w^{2}}, \phi_{1}(z, w)=\frac{z^{2}}{1-i w-\frac{1}{3} w^{2}}, \\
\phi_{2}(z, w)=\frac{\sqrt{\frac{13}{12}} z w}{1-i w-\frac{1}{3} w^{2}}, \phi_{3}(z, w)=\frac{\frac{\sqrt{3}}{3} w^{2}}{1-i w-\frac{1}{3} w^{2}}, g(z, w)=\frac{w-i w^{2}}{1-i w-\frac{1}{3} w^{2}} .
\end{gathered}
$$

It is equivalent to the proper polynomial holomorphic map $G$ from $\mathbb{B}^{2}$ into $\mathbb{B}^{5}$ :

$G(z, w)=\left(\frac{\sqrt{3}}{9}\left(-2+4 z+z^{2}\right),-\frac{\sqrt{6}}{9}\left(1+z+z^{2}\right), \frac{\sqrt{3}}{12}(5+3 z) w, \frac{\sqrt{6}}{6} w^{2}, \frac{\sqrt{13}}{12} i(1-z) w\right)$.

Proof: In fact, for the map $F$ given above, $e_{1}=-1, e_{2}=-\frac{1}{3}, c_{1}=\sqrt{\frac{13}{12}}, c_{3}=$ $\frac{\sqrt{3}}{3}$. From the proof of Theorem 3.1, the hyperplanes $H \subset \mathbb{C P}^{2}, H^{\prime} \subset \mathbb{C P}^{5}$ are defined by

$$
\begin{aligned}
& H: t=-y_{0} i w, \text { or } \frac{w}{t}=\frac{i}{y_{0}}, \\
& H^{\prime}: t^{\prime}=-\lambda_{4} z_{4}^{\prime}-\lambda_{5} w^{\prime}, \text { or }-\lambda_{4} \frac{z_{4}^{\prime}}{t^{\prime}}-\lambda_{5} \frac{w^{\prime}}{t^{\prime}}=1 .
\end{aligned}
$$

Here $y_{0}<0$ is a solution for $\left(y_{0}+1\right)^{3}-\frac{1}{3}\left(y_{0}+1\right)+\frac{2}{3}=0, \lambda_{4}=\frac{1}{c_{3}}\left[-\left(y_{0}-e_{1}\right)^{2}-e_{2}\right]=$ $-\frac{\left(y_{0}-e_{1}\right)^{2}+e_{2}}{\sqrt{e_{1} e_{2}}}$ and $\lambda_{5}=2 i y_{0}-e_{1} i$. Therefore $y_{0}=-2, \lambda_{4}=-\frac{2}{\sqrt{3}}$ and $\lambda_{5}=-3 i$. Thus we see that

$$
\begin{aligned}
& H: t=2 i w, \text { or } \frac{w}{t}=\frac{1}{2 i} \\
& H^{\prime}: t^{\prime}=\frac{2}{\sqrt{3}} z_{4}^{\prime}+3 i w^{\prime}, \text { or } \frac{2}{\sqrt{3}} \frac{z_{4}^{\prime}}{t^{\prime}}+\frac{3 i w^{\prime}}{t^{\prime}}=1 .
\end{aligned}
$$

Consider $\tilde{F}:=\rho_{5} \circ F \circ \rho_{2}^{-1}: \mathbb{B}^{2} \rightarrow \mathbb{B}^{5}$ where $\rho_{i}$ are the corresponding Cayley transformations. An easy computation shows that the projectivization of $\tilde{F}$, 
denoted by $\hat{\tilde{F}}$, is as follows:

$$
\begin{array}{r}
\hat{\tilde{F}}([z: w: t])=\left[z(3 t+w): 2 z^{2}: 2 i \sqrt{\frac{13}{12}} z(t-w):-\frac{2 \sqrt{3}}{3}(t-w)^{2}\right. \\
\left.: \frac{1}{3}\left(t^{2}+10 t w+w^{2}\right): \frac{1}{3}\left(13 t^{2}-2 t w+w^{2}\right)\right]
\end{array}
$$

and

$$
\begin{aligned}
& \hat{\tilde{H}}:=\hat{\rho}_{2}(H): t=\frac{1}{3} w, \\
& \hat{\tilde{H}}{ }^{\prime}:=\hat{\rho}_{5}\left(H^{\prime}\right): t^{\prime}=\frac{\sqrt{3}}{6} z_{4}^{\prime}+\frac{1}{2} w^{\prime} .
\end{aligned}
$$

We have $\hat{\tilde{H}} \subset \mathbb{C P}^{2}$ and $\hat{\tilde{H}}^{\prime} \subset \mathbb{C P}^{5}$, that satisfy the property:

$$
\begin{gathered}
\hat{\tilde{H}} \cap \overline{\mathbb{B}_{1}^{2}}=\emptyset, \hat{\tilde{H}}^{\prime} \cap \overline{\mathbb{B}_{1}^{5}}=\emptyset \text { and } \\
\hat{\tilde{F}}(\hat{\tilde{H}}) \subset \hat{\tilde{H}}^{\prime}, \quad \hat{\tilde{F}}\left(\mathbb{C P} \mathbb{P}^{2} \backslash \hat{\tilde{H}}\right) \subset \mathbb{C} \mathbb{P}^{5} \backslash \hat{\tilde{H}} .
\end{gathered}
$$

According to Lemma 2.1, let

$$
\begin{aligned}
\hat{\sigma}_{1}([z: w: t])= & {\left[\frac{2 \sqrt{2}}{3} w: z+\frac{t}{3}: t+\frac{z}{3}\right] } \\
\hat{\sigma}_{2}\left(\left[z_{1}^{\prime}: z_{2}^{\prime}: z_{3}^{\prime}: z_{4}^{\prime}: w^{\prime}: t^{\prime}\right]\right)= & {\left[\frac{1}{2}\left(z_{4}^{\prime}+\sqrt{3} w^{\prime}\right)-\frac{\sqrt{3}}{3} t^{\prime}: \frac{\sqrt{6}}{6}\left(w^{\prime}-\sqrt{3} z_{4}^{\prime}\right)\right.} \\
& \left.: \frac{\sqrt{6}}{3} z_{1}^{\prime}: \frac{\sqrt{6}}{3} z_{2}^{\prime}: \frac{\sqrt{6}}{3} z_{3}^{\prime}: t^{\prime}-\frac{\sqrt{3}}{6}\left(z_{4}^{\prime}+\sqrt{3} w^{\prime}\right)\right],
\end{aligned}
$$

then $\hat{\sigma}_{1} \in U(3,1)$ and $\hat{\sigma}_{2} \in U(6,1)$ with $\hat{\sigma}_{1}\left(\hat{\tilde{H}}_{\infty}\right)=\hat{\tilde{H}}$ and $\hat{\sigma}_{2}\left(\hat{\tilde{H}}^{\prime}\right)=\hat{\tilde{H}}_{\infty}^{\prime}$. The desired proper polynomial holomorphic map $G$ is thus induced by $\hat{\sigma}_{2} \circ \hat{\tilde{F}} \circ \hat{\sigma}_{1}$, which has the expression given in Proposition 3.3.

Remark 3.4: It may be interesting to notice that the map $G$ in Proposition 3.3 does not preserve the origin and is not equivalent to a map of the form $\left(G^{\prime}, 0\right)$. We do not know other examples of proper polynomial maps between balls of this type. 


\section{EXAmples of RATiOnal MAPS that ARE NOT EQUiVALENT TO POLYNOMIAL MAPS}

In this section, we apply Theorem 2.2 to construct examples of rational holomorphic maps which are not equivalent to proper holomorphic polynomial maps.

Example 4.1: Let $G(z, w)=\left(z^{2}, \sqrt{2} z w, w^{2}\left(\frac{z-a}{1-\bar{a} z}, \frac{\sqrt{1-|a|^{2}} w}{1-\bar{a} z}\right)\right),|a|<1$, be a map in $\operatorname{Rat}\left(\mathbb{B}^{2}, \mathbb{B}^{4}\right)$. Then $G$ is equivalent to a proper holomorphic polynomial map in $\operatorname{Poly}\left(\mathbb{B}^{2}, \mathbb{B}^{4}\right)$ if and only if $a=0$.

Proof: Indeed, we have

$$
\hat{G}=\left[(t-\bar{a} z) z^{2}:(t-\bar{a} z) \sqrt{2} z w: w^{2}(z-a t): w^{2} \sqrt{1-|a|^{2}} w:\left(t^{3}-\bar{a} t^{2} z\right)\right] .
$$

Suppose there exist hyperplanes $H=\left\{\mu_{1} z_{1}+\mu_{2} w+\mu_{0} t=0\right\} \subset \mathbb{C P}^{2}$ and $H^{\prime}=$ $\left\{\sum_{j=1}^{4} \lambda_{j} z_{j}^{\prime}+\lambda_{0} t^{\prime}=0\right\} \subset \mathbb{C P}^{4}$ such that

$H \cap \overline{\mathbb{B}_{1}^{2}}=\emptyset, H^{\prime} \cap \overline{\mathbb{B}_{1}^{4}}=\emptyset, \hat{G}(H \backslash \operatorname{Sing}(\hat{G})) \subset H^{\prime}, \hat{G}\left(\mathbb{C P}^{2} \backslash(H \cup \operatorname{Sing}(\hat{G}))\right) \subset \mathbb{C P}^{4} \backslash H^{\prime}$

Then

$$
\begin{array}{r}
\lambda_{1}(t-\bar{a} z) z^{2}+\lambda_{2}(t-\bar{a} z) \sqrt{2} z w+\lambda_{3} w^{2}(z-a t)+\lambda_{4} w^{2} \sqrt{1-|a|^{2}} w \\
+\lambda_{0}\left(t^{3}-\bar{a} t^{2} z\right)=\left(\mu_{1} z+\mu_{2} w+\mu_{0} t\right)^{3} \quad \forall[z: w: t] \in \mathbb{C P}^{2}
\end{array}
$$

Apparently $\lambda_{0} \neq 0$. Hence we can assume that $\lambda_{0}=1, \mu_{0}=1$. By comparing the coefficient of $z^{3}, w^{3}, w t^{2}, z t^{2}, z^{2} t, z w t, z^{2} w, z w^{2}, w^{2} t$, respectively, in the above equation, we get

$$
\begin{array}{r}
\mu_{1}^{3}=-\bar{a} \lambda_{1}, \mu_{2}^{3}=\lambda_{4} \sqrt{1-|a|^{2}}, 3 \mu_{2}=0,3 \mu_{1}=-\bar{a}, 3 \mu_{1}^{2}=\lambda_{1}, \\
6 \mu_{1} \mu_{2}=\sqrt{2} \lambda_{2}, 3 \mu_{1}^{2} \mu_{2}=-\sqrt{2} \lambda_{2} \bar{a}, 3 \mu_{1} \mu_{2}^{2}=\lambda_{3}, 3 \mu_{2}^{2}=-a \lambda_{3} .
\end{array}
$$

We then have $\lambda_{2}=\lambda_{3}=\lambda_{4}=\mu_{2}=0$. If $a \neq 0$, then $\mu_{1}, \lambda_{1} \neq 0$. From $\mu_{1}^{3}=-\bar{a} \lambda_{1}$ and $3 \mu_{1}^{2}=\lambda_{1}$, we get $\mu_{1}=-3 \bar{a}$. Since $3 \mu_{1}=-\bar{a}$, we get $\bar{a}=0$. This is a contradiction. Notice that when $a=0, F$ is a polynomial. By Theorem 2.2, we see the conclusion.

Example 4.2: Let $F\left(z^{\prime}, w\right)=\left(z^{\prime}, w z^{\prime}, w^{2}\left(\frac{\sqrt{1-|a|^{2}} z^{\prime}}{1-\bar{a} w}, \frac{w-a}{1-\bar{a} w}\right)\right)$ with $|a|<1$ be a map in $\operatorname{Rat}\left(\mathbb{B}^{n}, \mathbb{B}^{3 n-2}\right)$. Then $F$ has geometric rank 1 and is linear along each 
hyperplane defined by $w=$ constant. $F$ is equivalent to a proper polynomial map in $\operatorname{Poly}\left(\mathbb{B}^{n}, \mathbb{B}^{3 n-2}\right)$ if and only if $a=0$.

Proof: $\quad$ The projectivization of $F$ is

$$
\hat{F}=\left[t z^{\prime}(t-\bar{a} w): t w z^{\prime}: w^{2} \sqrt{1-|a|^{2}} z^{\prime}: w^{2}(w-a t): t^{2}(t-\bar{a} w)\right] .
$$

Assume $a \neq 0$ and suppose there exist hyperplanes $H \subset \mathbb{C P}$ and $H^{\prime} \subset \mathbb{C P}^{3 n-2}$ such that

$$
\begin{gathered}
H \cap \overline{\mathbb{B}_{1}^{n}}=\emptyset, H^{\prime} \cap \overline{\mathbb{B}_{1}^{3 n-2}}=\emptyset, \hat{F}(H \backslash \operatorname{Sing}(\hat{F})) \subset H^{\prime}, \\
\hat{F}\left(\mathbb{C P}^{n} \backslash(H \cup \operatorname{Sing}(\hat{F}))\right) \subset \mathbb{C P}^{3 n-2} \backslash H^{\prime} .
\end{gathered}
$$

Then

$$
\begin{gathered}
\lambda_{1}^{\prime} t z^{\prime}(t-\bar{a} w)+\lambda_{2}^{\prime} t w z^{\prime}+\lambda_{3}^{\prime} w^{2} \sqrt{1-|a|^{2}} z^{\prime}+\lambda_{n} w^{2}(w-a t)+\lambda_{0} t^{2}(t-\bar{a} w) \\
=\left(\mu_{0} t+\mu^{\prime} z^{\prime}+\mu_{n} w\right)^{3}
\end{gathered}
$$

for some $\lambda_{1}^{\prime}, \lambda_{2}^{\prime}, \lambda_{3}^{\prime}, \mu^{\prime} \in \mathbb{C}^{n-1}$ and $\lambda_{n}, \lambda_{0}, \mu_{0}, \mu_{n} \in \mathbb{C}$. Then $\lambda_{0}=\mu_{0}^{3} \neq 0$. We thus can assume at the beginning that $\lambda_{0}=\mu_{0}=1$.

Since there are no terms like $z_{j}^{3}(j<n)$ on the left hand side, we conclude that $\mu^{\prime}=0$. Thus we get

$$
\lambda_{n} w^{2}(w-a t)+t^{2}(t-\bar{a} w)=\left(t+\mu_{n} w\right)^{3} .
$$

Therefore $-\bar{a}=3 \mu_{n},-\lambda_{n} a=3 \mu_{n}^{2}, \lambda_{n}=\mu_{n}^{3}$ or $\mu_{n}=-\frac{\bar{a}}{3}$ and $\mu_{n}=-\frac{3}{a}$. This contradicts the assumption that $0<|a|^{2}<1$.

\section{REFERENCES}

[CD] D. Catlin and J. D'Angelo, A stabilization theorem for Hermitian forms and applications to holomorphic mappings, Math Research Letters, 3(1996), 149-166.

[CJX] Z. Chen, S. Ji and D. Xu, Rational holomorphic maps from $\mathbb{B}^{2}$ into $\mathbb{B}^{N}$ with degree 2. Science in China, 49(2006), 1504-1522.

[DA] J.P. D'Angelo, Proper rational mappings, Proceedings of the Mittag-Leffler Institute on Several Complex Variables, Mathematical Notes 38(1993), Princeton University Press, Princeton, New Jersey, 227-244.

[Fa] J. Faran, The linearity of proper holomorphic maps between balls in the low codimension case, J. Diff. Geom. 24(1986), 15-17.

[Fr] F. Forstneric, Proper holomorphic maps from balls, Duke Math. J. 53, 1986, 427-441.

$[\mathrm{Hu}] \mathrm{X}$. Huang, On a linearity problem of proper holomorphic mappings between balls in complex spaces of different dimensions, J. Diff. Geom. 51(1999), 13-33. 
[HJX] X. Huang, S. Ji and D. Xu, A new gap phenomenon for proper holomorphic mappings from $\mathbb{B}^{n}$ to $\mathbb{B}^{N}$, Math Research Letter, 3(2006), no. 4, 509-523.

\section{James Faran}

Department of Mathematics

The State University of New York at Buffalo, Buffalo, NY 14260, USA

E-mail: jjfaran@buffalo.edu

\section{Xiaojun Huang}

Department of Mathematics

Rutgers University, New Brunswick, NJ 08903, USA

E-mail: huangx@math.rutgers.edu

Shanyu Ji

Department of Mathematics

University of Houston, Houston, TX 77204, USA

E-mail: shanyuji@math.uh.edu

Yuan Zhang

Department of Mathematics

Rutgers University, New Brunswick, NJ 08903, USA

E-mail: yuanz@math.rutgers.edu 